\title{
Obtaining Impactful Feedback from Students: A Continuous Quality Improvement Approach to Enhance the Quality of Students' Feedback
}

\author{
Dujeepa D. Samarasekera ${ }^{1}$, Yeo Su Ping ${ }^{2}$, Tan Chay Hoon ${ }^{3}$
}

\begin{abstract}
Academic institutions collect student feedback to improve their curriculum and teaching quality. At the Yong Loo Lin School of Medicine (YLLSoM), National University of Singapore (NUS), the feedback is also used to disburse funds to clinical teaching sites as well as to make decisions on faculty performance. There are studies on the benefits and uses of measurement tools (e.g. student ratings) to evaluate university courses, however, studies evaluating the process of effecting changes to the system are few. In this study, the authors discuss the limitations of the student feedback system/processes and the way new changes were instituted to overcome these limitations at YLLSoM, NUS. This study highlights the importance of constantly reviewing the feedback mechanism to obtain better inputs for improving the learning environment. Our findings are particularly useful for the training of clinical teachers in Asian and new medical schools, who can learn from our strengths to meet the required standards.
\end{abstract}

Keywords: Student feedback, stakeholder engagement, continuous quality improvement, curriculum development,

\section{Introduction}

Obtaining timely feedback from students is necessary to implement continuous improvements to the curriculum and the teaching-learning environment. The significance of student feedback to teachers is highlighted in studies (Watsons, 2003) as it provides a major source of evidence for examining teaching quality and effectiveness (Richardson, 2005). This is particularly important since medical teachers make indispensable contribution in providing students with support, academic feedback and directions (Stalmeijer et al., 2010).

\footnotetext{
${ }^{1}$ Director, Medical Education Unit

${ }^{2}$ Executive, Medical Education Unit

${ }^{3}$ Medical Education Unit Associate and Associate Professor, Department of Pharmacology

Yong Loo Lin School of Medicine, National University of Singapore, Singapore

Corresponding author:

Dujeepa D Samarasekera, Yong Loo Lin School of Medicine, National University of Singapore, 1E Kent Ridge Road, NUHS Tower Block Level 11, Dean's Office, Singapore 119228.
}

E-mail:meddds@nus.edu.sg
There are some concerns with the ratings students give on the quality of teaching or the student's perceived feelings about the effectiveness of a course or program. For example, some feel that tutors who are more humorous/friendly and expressive tend to receive higher ratings. Although teachers who utilise interesting presentations may get higher ratings, it may actually be due to the fact that these lectures excite the students, thus capturing their attention and stimulating their learning (Cashin, 1995). Further, some have questioned the influence of extraneous factors on the ratings. However, Keane \& Labhrainn (2005) report that teacher's attributes such as personality and teaching experience, student factors (e.g. age, ethnicity and grade average), and course variables such as the size and timing of the class in general do not affect the students' perception of the tutor's quality of teaching/effectiveness (Keane \& Labhrainn, 2005). In addition, a factor may only be considered to bias the reliability and validity (negatively or positively) of student ratings, if it demonstrates substantial relationships with the data obtained from student feedback, and it does not encourage student learning (Centra, 2003). Several studies argue that the majority 
of these extraneous factors do not influence students' ratings since they do not meet these criteria (Remedios \& Lieberman, 2008; Centra, 2008).

Additionally, recent studies have shown that student ratings are "multidimensional, reliable and stable, valid and relatively unaffected by many variables hypothesized as potential biases to it" (Spencer \& Schmelkin, 2002; Williams \& Brennan, 2003). In fact, student feedback on teachers have been found to be more closely correlated and reliable than information obtained through peer evaluation by other teaching staff (Richardson, 2005).

Feedback provides insights to the teachers on whether the curriculum/module has met the intended outcomes and/or requires improvements. Moreover, teaching methods can be assessed and weaknesses rectified, especially when major discrepancies exist between a teacher's self-assessment and students' feedback (Stalmeijer et al., 2010).

\section{Background to the Study}

The undergraduate medical program at Yong Loo Lin School of Medicine (YLLSoM), National University of Singapore (NUS) is a five-year course, where students learn basic medical sciences in the first two years. Subsequently, they are posted to clinical training sites which include restructured hospitals and other training sites such as polyclinics which are government subsidised first contact care clinics, general practitioner clinics and community hospitals across Singapore to develop clinical skills necessary for future practice. Voluntary student feedback regarding the effectiveness of the teachinglearning activities and the teachers involved is regularly obtained by YLLSoM. The feedback data is used by the school for many purposes. Apart from using the data for program enhancements or revision and teacher selfimprovement, the clinical posting scores are also used to disburse funding allocated for teaching medical students by the Ministry of Health. The teacher scores are also used for department ranking purposes of teachers during their annual performance evaluations and to determine salary bonuses, promotions or for tenure purposes. Hence, matters pertaining to feedback scores are sensitive issues as they have a major impact on faculty members, departments and clinical training sites. Any changes or improvements to the student feedback process had to be managed in a transparent and collaborative manner with all the key stakeholders.

Following meetings with students, Dean's office academic staff and those involved in administering feedback, the following key challenges were documented prior to changes to the feedback process in 2009.

\section{Questionnaire Fatigue}

Unlike other NUS faculties where feedback is conducted approximately once a semester at the end of their modules, medical students were sometimes required to complete feedback forms every two-three weeks, with over ten questions per teacher for more than ten tutors on each occasion. Consequently, students frequently shunned the feedback process which led to a lower response rate. This is a common challenge in many other institutions, which could lead to an unrepresentative conclusion, since those who responded might have a stronger opinion of certain issues. Generally, a response rate of $60 \%$ and higher is recommended, and data with a response rate of less than $30 \%$ should be analysed with extreme care (Keane \& Labhrainn, 2005).

\section{Nature of the Forms}

Obtaining specific feedback for learning was a challenge. This was because firstly, a common feedback form was used regardless of the nature of training (i.e. pre-clinical or clinical) or the lesson type (e.g. clinical posting or a learning module) prior to 2009. This is a common problem - questionnaires may be too generic and lack descriptiveness and specificity (Stalmeijer et al., 2010). If there is inadequate link between how tutors are evaluated and what they are teaching, they may not be motivated to make any change (Baker et al., 2010). Secondly, the Likert scale lacked clarity and standardization. Different students had their own definitions of what selected choices such as 'Agree' or 'Disagree' meant to them.

\section{Time-Consuming and Error-Prone}

Students completed one hardcopy form for the teachers involved in a posting or module as well as another on the teaching-learning activities experienced. The collection of feedback from nearly 1300 medical students required many steps as well as processing and analysis of this data (e.g. from collecting and receiving the forms, scanning, analysis, 
uploading, etc.). The long processing time which sometimes took 6-12 months hampered prompt implementation of corrective actions to the learning environments. Delay in processing feedback data leading to delays in implementing relevant changes to the learning environment has been documented in other studies (Harvey, 2001). Delays were usually due to data discrepancies such as errors when entering data to the system during tagging students to specific teachers.

In view of these challenges, as well as the curricula revisions effective from AY2006 onwards to both the pre-clinical and clinical years of learning, in-depth discussions were held involving key stakeholders to improve the feedback system.

To circumvent the above challenges and limitations, YLLSoM, Dean's Office Quality Improvement team instituted revisions from 2008 onwards to the student feedback collection process. The main focus of these reforms were to improve clarity of feedback items, institute a systematic process in collection, analysis and reporting with clearly laid down standard operating procedures leading to system efficiency.

\section{Reforms to the Feedback System over the last 4 Years (2008 onwards)}

Some major improvements are discussed below.

\section{Feedback Form Revisions}

\section{(1a) Format}

In AY11/12 Semester 2, departments involved in the pre-clinical years started using an online system. Following positive responses, this trial was expanded to the clinical postings at the National University Hospital in 2012. Reasons behind the shift include:

- allowing students to provide feedback at their convenience, rather than at the end of lectures (they may feel pressurized as the tutor to be evaluated is present),

- reducing the processing time and minimizing human errors,

- reducing the use of paper, to save cost and benefit the environment.

\section{(1b) Close-ended questions' scale}

As explained previously, the Likert scale lacked standardization and was overlygeneric, making it hard to associate the scale with what the question was asking. For questions like 'The teacher provided timely and useful feedback', ratings like 'neutral' may be ambiguous. Hence, descriptors were introduced since AY11-12. Choices were more elaborate and specific to each question. Choices like 'frequently' and 'routinely' certainly make more sense than 'neutral' in this example.

\section{(1c) Number of Questions/ltems}

The number of questions had been gradually reduced over the years (see Table 1), which was partly due to changes made to the questions' focus. Rather than rating three separate questions -'focused on the learning objectives', 'integration of the basic sciences with clinical sciences', and 'overall teaching effectiveness', these were combined into one question on overall effectiveness.

\section{1d) Focus/Types of Questions}

In 2009, different forms were introduced for pre-clinical and clinical lessons, which increased the specificity and allowed teachers to better assess their performance in different settings (module versus posting). Following a medical curriculum review where new teaching tools were added to the syllabus (e.g., Mini Clinical Exercise), questions related to these activities were included in the clinical forms. Since AY12-13, questions irrelevant to a department in the posting forms were removed, which increased the relevance of the feedback.

Furthermore, there was a shift in the question focus for some forms. We will briefly examine the preclinical module and clinical posting forms.

\section{Preclinical Module}

In AY11-12, several relatively less important questions e.g. 'The grade I am likely to get for the module is ', were removed from the forms. Till AY10-11, most questions were program specific/ on effectiveness, e.g. 'objectives were made clear to students' and 'module was taught with sufficient clinical relevance'. However, in AY11-12, there was a shift towards questions on the learning process, e.g. 'designated teachers were accessible during scheduled training sessions to provide effective teaching'. In AY12-13, the emphasis was almost entirely on the student's learning experience. Open-ended (on module's strengths and improvements), and close-ended questions ask the students to rate their overall learning experience derived from the learning processes e.g. their experience with the teaching-learning activities. 
Clinical Posting

In AY09-10, there was a mixture of the following types of questions:

- Program-specific: e.g. 'received clear learning objectives'.

- Processes: e.g. 'tutor mentored me throughout my posting', 'tutor personally observed me performing physical examination'

- Learning experience e.g. "I would rate the program as
In AY11-12, while the number of programrelated questions was kept relatively similar, it was made more specific to different classroom settings e.g. 'learning activities were wellaligned to the posting objectives' in bedside tutorials, ambulatory teaching and ward work training (to be rated separately). Next, questions on learning processes and experience (e.g. 'clinical care-team experience... helps me develop essential clinical skills....') were greatly reduced and increased respectively.

Table 1: Question reduction over the years

\begin{tabular}{lcccc}
\hline & \multicolumn{4}{c}{ Number of Questions } \\
\cline { 2 - 5 } Academic Year & $\begin{array}{c}\text { Pre-Clinical } \\
\text { Module }\end{array}$ & $\begin{array}{c}\text { Pre-Clinical } \\
\text { Teaching }\end{array}$ & Clinical Posting & Clinical Teaching \\
\cline { 2 - 5 } AY09-10 & 9 & 11 & 18 & 12 \\
AY11-12 & 6 & 7 & 9 & 8 \\
AY12-13 & 3 & 3 & At most 9 & At most 8 \\
Reduction Since AY 09-10 & $67 \%$ & $72 \%$ & At least 50\% & At least 33\% \\
\hline
\end{tabular}

\section{Feedback Processes}

In 2009, Standard Operating Procedures (SOPs) for the hardcopy feedback system were drafted, which contained information such as the timeline for processes (e.g. deadline for tagging of teachers) and the parties involved. With the commencement of online feedback, another set of SOPs was drawn up in 2012.

\section{Study's Objectives}

Our aim was to identify the impact of changes implemented to the student feedback system and to obtain stakeholders' views of the process with a view to further improvements.

\section{Methodology}

Approval from the Institutional Review Board, National University of Singapore, was obtained.

The interviewees included those involved in the administration of feedback (e.g. data collection) and those who oversee the feedback process at the respective hospitals and the Dean's Office, as well as key student leaders. This allowed us to obtain a more comprehensive understanding of the entire process from people at the collection, collation and analysis stages all the way to the implementation phase to "complete the loop".
Those who are not involved in feedback administration or overseeing the process were excluded. A total of 6 administrative staff, 2 students and 13 academic staff were interviewed in this study. Semi-structured interviews were conducted by one of the investigators. The interview questions were refined after a pilot study comprising the following broad themes: 1) Strengths and limitations of the old and the new feedback systems, 2) Challenges and recommendations.

Furthermore, minutes of the student class representative meetings, which are held every two to three months, were analysed and items relevant to student feedback were extracted.

The students' feedback data for the past 2 years were also analysed and comments on the feedback process, issues, strengths and student suggestions were also collated.

All data obtained (interviews, feedback, meetings) were first categorized into the following thematic groups:

- Old feedback system- Strengths and limitations

- New feedback system- Strengths and limitations

- Challenges

- Recommendations to further optimize the system 
Data obtained from the interviews was categorized into the following three occupation categories: administrative, academics and student. Within each category, data was further clustered thematically. For example, comments by interviewees that reflected the convenience of online feedback were grouped together. Finally, the data obtained from these three sources were triangulated to evaluate the areas of agreement and divergence between different themes and data sources. This was followed by the identification of the areas for further improvements.

\section{Results}

\section{Interviews}

The key findings are presented below.

\section{Strengths of the old feedback system}

All three groups (academics, administrative and students) felt that hardcopy forms tend to record a higher response rate as students usually complete and submit them at the end of the lessons.

\section{Limitations of the old feedback system}

Three major points were brought up by all groups. Firstly, the older forms contained items that were ambiguous and unspecific. Secondly, the problem of questionnaire fatigue was prevalent as the forms had items sometimes not relevant to the learning setting. Thirdly, the issue of recall bias, as students may not remember the teaching-learning events when feedback was collected at the end of their postings/modules.

Both academics and administrative personnel found the older system too tedious, involving manual stages and required considerable manpower resources. This was exacerbated by inadequate adherence to guidelines from departments and frequent last-minute changes to teachers or students tagged for feedback which contributed to high incidences of errors. The academic staff and students also perceived that the information collected may be inaccurate and subjective.

\section{Strengths of the new feedback system}

In general, the changes were positively received. All three groups were pleased with the increased specificity and relevance of the newer questions, which provided more useful and informative feedback to the teaching staff to improve the standards of teaching and the curriculum. The descriptors were also considered to be more standardized.
The generation of the reports was felt to be faster, within 4 to 6 weeks of the teachinglearning session, with the changes in place according to the academics and administrative staff. Also, findings revealed that questionnaire fatigue decreased after the reduction in questions (by the academics group), and that the introduction of online feedback significantly reduced paper usage. To the administrative staff involved in the feedback collection, the online system has helped them/and the departments to monitor the response rates.

\section{Limitation of the new feedback system}

Despite the many improvements, several limitations were identified. Of major concern among all three groups was the lower student response rate. This was attributed to the large number of tutors in the system to be evaluated. The academics and students still felt that the time lag of generating the reports from feedback collection, even though vastly improved, hindered the rapidity of corrective actions to be made to their teaching style or to the curriculum. They also felt that despite the huge amount of time and effort spent, the reports may be affected by "popularity contest" /human emotion effects and the non-ideal feedback collection timing (recall bias).

The academics argued that the coordination and control of the feedback process was overly centralized, which occasionally lead to inefficiencies. They preferred questionnaire items to be more specific and flexibility for the individual hospitals or module coordinators to change according to learning relevance. To give the new/inexperienced tutors a chance to improve, the academics commented that the tutor's appraisal should be detached from the outcome of the feedback process. On the students' side, some voiced displeasure over the non-user friendly online feedback system.

\section{Challenges}

All three groups acknowledged that it is a huge challenge to get students to complete online feedback. The low responses may be due to inadequate availability of computer rooms as highlighted by some academics and administrative staff or the non-optimal feedback window period, an area highlighted by academics and students.

With regards to the feedback process, the majority of the administrative and academic staff felt that understaffing is prevalent, often with heavy reliance on one person performing a specific role. This could be a result of the high turnover rate caused by fatigue which 
partly arises from the need to remind the departments to follow the guidelines.

\section{Recommendations}

One of the key objectives of this study was to gather opinions on how the feedback system can be further refined.

The most important recommendations were those highlighted by members from all three groups: (1) Engage the students, let them know why they should give feedback, (2) Introduce incentives/penalties to increase the response rate, (3) Design simpler forms with more qualitative questions, and phrase the questions differently to emphasise the importance of their feedback to the juniors, (4) Implement the online feedback system across all the hospitals, (5) Give the Associate Deans of Hospitals and key education leads/teachers a bigger role in the feedback process, e.g. to design their own questions.

The second category of suggestions contains pointers brought up by members from two groups. For example, the academics and administrative staff proposed: (1) More support/coordination from the departments, and that they need to follow the instructions properly, (2) Consider other indicators apart from student feedback to evaluate the curriculum and teaching. The academics and students felt that: (1) Feedback should be collected at a better time e.g. end of an assessment or a series of lectures for increased accuracy and (2) Longer window period for students to give their feedback. Finally, some participants from the student and administrative groups requested the following changes to be made to improve the system: (1) Better IT support/ more convenient IT platforms for feedback e.g. MedSpace and (2) Introduce more focus group discussions to get more timely and informative qualitative comments.

\section{Discussion}

From the findings, four key areas were identified for further development. These are discussed below.

\section{User-friendly Format}

Feedback in a format that is user-friendly is key to an efficient feedback system as it influences the response rates and data accuracy. Indeed, we found that students place great emphasis doing surveys on platforms deemed convenient to them. This is evident from the majority of the students who preferred the questionnaires to be hosted on various IT platforms (e.g. iPad and mobile phones), and e-Learning portals (e.g. IVLE and MedSpace). Next, while a switch to the online feedback system was expected to bring about many benefits such as students providing feedback at their own discretionary time (Johnson, 2003), our data suggested that this was not achieved. One possible explanation could be that students felt the online interface tedious, as it requires switching between different pages many times. Again, this attests to the fact that user-friendly level of a feedback system can influence students' responses.

A study by Brigham Young University (BYU) in the United States concluded that the length of the feedback forms was not a major consideration for most students when deciding whether to complete the questionnaires (Johnson, 2003). However, our respondents agreed that the revamped and shorter forms reduced questionnaire fatigue, and in fact, proposed a further reduction in the number of questions. This may be attributed to the different frequencies of feedback collection, as our students have to complete several surveys every 2-4 weeks. Hence, the survey length takes on more significance here.

\section{Feedback Forms with the Right Questions}

As Stalmeijer et al. (2010) pointed out, some questionnaires may be overly generic and lack the required descriptiveness and specificity. When inadequate link exists between how tutors are evaluated and how they teach, they may not feel motivated to make any change (Baker et al., 2010), rendering the feedback process useless. In view of this, we revised the questions over the years, to allow information more useful to the academic staff to be collected. Indeed, our findings are consistent with his arguments, as interviewees praised the increased specificity and relevance of questions. The impact is also evident from the improvements in many tutors' teaching abilities.

\section{An Efficient Team of Feedback Administrators}

Before the changes were administered to the system, the hardcopy feedback forms and tedious processing steps which involved massive manpower had greatly hindered the feedback process. With the revamps, in particular the SOPs and the switch to an online feedback system, we found that our administrative staff work efficiency and quality 
greatly increased. Indeed, less time was utilized for processing, with an increased ease of administration and more complete data sets, which are benefits commonly associated with an online feedback system (Johnson, 2003). Consequently, the issue of understaffing was mitigated, allowing staff to focus more on other aspects of the feedback process, which led to a shorter report generation time.

\section{Closing the Feedback Loop}

As Watsons (2003) describes, it is important to close the feedback loop, a step often found lacking in many feedback systems. Our analysis supports this argument, as students' nonchalant attitude towards feedback and low response rates are largely because they felt their inputs were not used to improve the educational environment. Studies related to student ratings have suggested that students' belief that feedback data are utilized for major decisions involving the teaching faculty and curriculum plays a big part in influencing their response rates (Ballantyne, 1999). While we have been closing the loop through the implementation of corrective actions based on their suggestions, we felt that it was crucial to step up efforts to engage students more, and let them know the significance of their inputs. This is consistent with what many other institutions are doing, e.g. BYU, which shares feedback results through student meetings, emails and webpage (Johnson, 2003). All in all, students want their voices to be heard, and to know that their responses make a difference to their educational environment.

\section{Proposed Actions}

Reforms to the feedback system have led to improvements to the clarity of forms providing more specific and informative data to the stakeholders as well as efficacy to the overall feedback process. However, the study also revealed concerns with regard to the low response rates from students and the possibility of this process being manipulated for personal gains. These are common limitations encountered in many other medical schools globally. Another feature of this study is the engagement of all stakeholders in a sensitive manner to effect change effectively in a challenging educational process at a large institution.

With these findings, the feedback process at YLLSoM will be further enhanced and refined in several areas during future academic terms. First, an online portal to disseminate results of the feedback was established. This allowed individual teachers, key undergraduate program coordinators and directors as well as the Heads of Department to have a 24/7 online dashboard to access the information. This system will be linked in the future to a new online and mobile enabled student feedback portal so that the analysis will be automated and transferred directly to the online dashboard.

The SOPs have been further refined as well as simplified, and subsequently disseminated to the individual department's administrative staff. The SOPs have also been uploaded to the faculty's online portal, which can be accessed anytime by the staff. Furthermore, the feedback forms have been further modified to have only four items focusing on the overall effectiveness of the posting and the teacher. The feedback team is also partnering with the students' representative body (MedSoc), to identify areas for improvement in the feedback process. It is hoped that by engaging the students more, better quality comments and higher response rates can be obtained for the benefit of the teaching faculty. Areas to anchor include the feasibility of using social media like Facebook and Twitter to collect feedback, and to get their response immediately after a teaching activity. Student feedback using iPad and Android-based devices has been rolled out for all students.

The possibility of allowing flexibility to include more items in the feedback forms by key educational leads at various sites is also explored. Moreover, the analysis process would be decentralized where online access is not extended presently, so that distance clinical training sites at other restructured hospitals in particular would have real-time results, thus reducing the lag-time so that teaching staff can make rapid improvements. While there are plans for all the restructured hospitals to switch to the online feedback system, this is not expedited due to context specific local issues like IT challenges arising from developing hospital-specific firewalls, which need to be addressed first.

\section{Conclusion}

In summary, our study has demonstrated the importance of reviewing important educational system processes in a regular and appropriate manner to effect change, so that the teachers can improve the curriculum and their teaching. We have also shared our approach in tackling several key challenges, which could be highly useful to the other medical schools. 
As with many other studies, several limitations exist. Perhaps a mixed-method, combining the qualitative research in this project with quantitative methods like student surveys could provide more insights into the students' perspective. However, we decided against this, as several sources of student data, including the interviews and findings from the focus groups were available. Moreover, we did not wish to add to the number of feedback forms students were already required to submit, which could potentially result in extremely unfavourable and biased responses.

Future research could evaluate the impact of the recommendations proposed in this paper, and shared with the wider community. Additionally, we hope this paper will spur similar research in other institutions, and provide opportunities for potential discussion and collaborations.

\section{Acknowledgements}

This research study was funded by the Teaching Enhancement Grant from the National University of Singapore's Centre for Development of Teaching and Learning. We express our gratitude to the Continuous Quality Improvement Team from Yong Loo Lin School of Medicine Dean's Office for their assistance and inputs to this study.

Declaration of interest: The authors report no conflicts of interest.

\section{References}

Baker, E. L., Paul, E. B., Hammond, L.D., Haertel, E., Ladd, H.F., Linn, R.L., Ravitch, D., Rothstein, R., Shavelson, R.J., \& Shepard, L.A. (2010) Problems with the use of student test scores to evaluate teachers (Economic Policy Institute Briefing Paper Number: 278) [Online] Available from http://www.epi.org/publication /bp278/[Accessed 2 October, 2012].

Ballantyne, C. (1999) Improving university teaching: Responding to feedback from students. In Zepke, N., Knight, M., Leach, L., and Viskovic, A. (Eds.), Adult learning cultures:challenges and choices in times of change, Wellington, Australia: WP Press (p155-165).

Cashin, W.E. (1995) Student ratings of teaching: The research revisited. [Online] Available from http://ideaedu.org/sites/default/files/ldea_Paper_ 32.pdf [Accessed July 2, 2013].
Centra, J.A. (2003) Will teachers receive higher student evaluations by giving higher grades and less course work? Research in Higher Education, 44, 5, pp. 495-518.

Centra, J.A. (2008) Differences in student ratings of instruction: Is it bias? Paper presented at the 88th annual meeting of the American Educational Research Association, New York.

Harvey, L. (2001) Student feedback: A report to the higher education funding council for England. (Centre for Research into Quality) [Online] Available from http://www.bcu.ac.uk/cra /publications/studentfeedback.pdf [Accessed October 1, 2012].

Johnson, T.D. (2003). Online Student Ratings: Will Students Respond? New Directions for Teaching and Learning (Special Issue: Online Student Ratings of Instruction), 96, pp. 49-59.

Keane, E. \& Labhrainn, I.M. (2005) Obtaining student feedback on teaching and course quality (Centre for Excellence in Learning \& Teaching, Briefing Paper 2) [Online] Available from http://www.nuigalway.ie/celt/documents/evaluati on_ofteaching.pdf [Accessed October 02, 2012].

Remedios, R. \& Lieberman, D.A. (2008) I liked your course because you taught me well: The influence of grades, workload, expectations and goals on students' evaluations of teaching, British Educational Research Journal, 34, 1, pp. 91-115.

Richardson, J.T.E. (2005) Instruments for obtaining student feedback: a review of the literature, Assessment \& Evaluation in Higher Education, 30, 4, pp. 387-415.

Spencer, K.J. \& Schmelkin, L.P. (2002) Student perspectives on teaching and its evaluation, Assessment and Evaluation in Higher Education, 27, pp. 397-409.

Stalmeijer, R. E., Dolmans, D. H., Wolfhagen, I. H., Peters, W. G., van Coppenolle, L., \& Scherpbier, A. J. (2010) Combined student ratings and self-assessment provide useful feedback for clinical teachers, Advances in Health Sciences Education, 15, 3, pp. 315-328.

Watsons, A. (2003) Closing the feedback loop: Ensuring effective action from student feedback, Tertiary Education and Management, 9, pp. 145-157.

Williams, R. \& Brennan, J. (2003) Collecting and using student feedback on quality and standards of learning and teaching in Higher Education. Bristol, UK. [Online] Available from http://www.heacademy.ac.uk/assets/documents/ tla/collecting_and_using_student_feedback.pdff Accessed September 30, 2012]. 